\title{
LONG-TERM CONSEQUENCES OF PRE-TERM BIRTH
}

\author{
B.M. Hansen \\ Department of Neonatology, University Hospital of Copenhagen, Copenhagen, Denmark
}

In the last decades many well conducted follow up studies have established that ex-preterm children have an increased risk of a wide range of developmental problems appearing in childhood; intellectual and motor impairments, school difficulties, behavioral and emotional problems. Most of these studies have focused on the development of children born very preterm $(\mathrm{GA}<32$ weeks) and extremely preterm $(\mathrm{GA}<28$ weeks $)$.

The number of longitudinal studies of ex-preterm individuals at adult age is more limited. These studies are difficult to conduct. Selection bias due to loss of follow up may be a major problem. The development of moderate preterm infants (GA: 32-36 weeks) is not well investigated compared to the children born with a lower gestational age.

In the last years register based studies from the Scandinavian countries have been reported supplementing the data on the long-term consequences of pre-term birth. High follow up rates and the ability to include a large number of individuals enable these studies to investigate a wide range of long term outcome.

Data on long-term outcome from danish register based studies including the whole spectrum of prematurity will be presented. The data show an increased risk of developmental problems for all preterm infants, there seems to be a dose-respons curve with an increasing risk for decreasing gestational age. Further it seems that the majority of ex-premature individuals are well integrated in society as adults and that they do better than expected compared to the wide range of problems described in childhood. 